\title{
Quantum Repeaters using Coherent-State Communication
}

\author{
Peter van Loock ${ }^{1,2}$, Norbert Lütkenhaus ${ }^{3}$, W. J. Munro ${ }^{2,4}$, and Kae Nemoto ${ }^{2}$ \\ ${ }^{1}$ Optical Quantum Information Theory Group, Institute of Theoretical Physics I and Max-Planck Research Group, \\ Institute of Optics, Information and Photonics, Universität Erlangen-Nürnberg, Staudtstr. 7/B2, 91058 Erlangen, Germany \\ ${ }^{2}$ National Institute of Informatics, 2-1-2 Hitotsubashi, Chiyoda-ku, Tokyo 101-8430, Japan \\ ${ }^{3}$ Institute for Quantum Computing, University of Waterloo, Canada \\ ${ }^{4}$ Hewlett-Packard Laboratories, Filton Road, Stoke Gifford, Bristol BS34 8QZ, United Kingdom
}

\begin{abstract}
We investigate quantum repeater protocols based upon atomic qubit-entanglement distribution through optical coherent-state communication. Various measurement schemes for an optical mode entangled with two spatially separated atomic qubits are considered in order to nonlocally prepare conditional two-qubit entangled states. In particular, generalized measurements for unambiguous state discrimination enable one to completely eliminate spin-flip errors in the resulting qubit states, as they would occur in a homodyne-based scheme due to the finite overlap of the optical states in phase space. As a result, by using weaker coherent states, high initial fidelities can still be achieved for larger repeater spacing, at the expense of lower entanglement generation rates. In this regime, the coherent-state-based protocols start resembling single-photon-based repeater schemes.
\end{abstract}

PACS numbers: 03.67.Lx, 42.50.Dv, 42.25.Hz

\section{INTRODUCTION}

In long-distance, classical communication networks, signals that are gradually distorted during their propagation through a channel are repeatedly recreated via a chain of intermediate stations along the transmission line. For instance, optical pulses traveling through a glass fiber and being subject to photon loss can be reamplified at each repeater station. Such an amplification is impossible, when the signal carries quantum information. If a quantum bit is encoded into a single photon, its unknown quantum state cannot be copied along the line [1, 2]; the photon must travel the entire distance with an exponentially decreasing probability to reach the end of the channel.

The solution to the problem of long-distance quantum communication is provided by the so-called quantum repeater [3, 4]. In this case, prior to the actual quantum-state communication, a supply of standard entangled states is generated and distributed among not too distant nodes of the channel. If sufficiently many of these imperfect entangled states are shared between the repeater stations, a combination of entanglement purification and entanglement swapping extends this shared entanglement over the entire channel. Through entanglement swapping [5], the entanglement of neighboring pairs is connected, gradually increasing the distance of the shared entanglement. The entanglement purification [6, 7] enables one to distill (through local operations) a high-fidelity entangled pair from a larger number of lowfidelity entangled pairs, as they would emerge after a few rounds of entanglement swapping with imperfect entangled states, or even at the very beginning after the initial, imperfect entanglement generation and distribution.

Current implementations for quantum communication, in particular, quantum key distribution, are limited by a distance of about $200 \mathrm{~km}$. In principle, one could go be- yond this distance using a quantum repeater. However, the issue of actually realizing a quantum repeater protocol is rather subtle, even for not too long distances. In particular, the subroutines of entanglement distillation and swapping require advanced local quantum logic including, for instance, two-qubit entangling gates; moreover, a sufficient quantum memory is needed such that local measurement results can be communicated between the repeater stations [8]. Nonetheless, various proposals exist, of which the most recent ones are based on the nonlocal generation of atomic (spin) entangled states, conditioned upon the detection of photons distributed between two neighboring repeater stations. The light, before traveling through the communication channel and being detected, is scattered from either individual atoms, for example, in form of solid-state single photon emitters [9, 10], or from an atomic ensemble, i.e. a cloud of atoms in a gas [11]. In these heralded schemes, typically, the fidelities of the initial entanglement generation are quite high, at the expense of rather small efficiencies. Other complications include interferometric phase stabilization over large distances [12, 13, 14] and the purification of atomic ensembles. Yet some elements towards a realization of the protocol in Ref. [11] have been demonstrated already [15, 16, 17]. Further theoretical results were presented very recently 18], improving the scheme of Ref. [11].

In this paper, we will extend our previous results on the so-called hybrid quantum repeater [19, 20]. This approach to long-distance quantum communication is somewhat different from those mentioned above. It relies on atom-light entanglement which becomes manifest in quantum correlations between a discrete spin variable and a continuous optical phase quadrature rather than a discrete single-photon occupation number. An optical pulse in a coherent state of about $10^{4}$ photons is subject to a controlled phase rotation (achieved through 
dispersive, CQED-type interactions), conditioned upon the state of the atom. After propagating to the nearest neighboring repeater station and a further interaction with a second spin at that station, the light field is measured via homodyne detection and an imperfect entangled two-qubit state is nonlocally prepared between the two repeater stations through postselection. Finally, the same dispersive light-matter interactions are exploited to achieve the local quantum gates ("qubus computation" $[21,22]$ ) needed for entanglement purification and swapping.

The two main advantages of the hybrid repeater protocol, distinct from the single-photon-based schemes, are the high success probabilities for postselection in the entanglement generation step and the intrinsic phase stabilization provided through reference pulses propagating in the same channel as the probes. However, these assets are at the expense of rather modest initial fidelities of the entangles states and high sensitivity to photon losses and noise in the optical channel. In fact, distances between repeater stations beyond $10-20 \mathrm{~km}$ turn out to be impossible with the current proposal; the decoherence effect (a damping of the off-diagonal terms of the two-qubit density matrix after postselection) exponentially grows with distance such that only smaller mean photon numbers lead to a sufficient degree of entanglement; however, the less intense coherent states are less distinguishable, hence resulting in a further decrease of fidelity through postselection errors. A good trade-off between these competing sources of errors is only possible for not too large distances.

The analysis here will provide a possible solution to the distance limitation. This is particularly important, as the typical repeater spacing in existing classical communication networks is of the order of 50-100 km and thus incompatible with the current hybrid repeater protocol. The distance limit can be overcome by completely eliminating one source of errors, namely that which stems from the finite overlaps of the phase-rotated coherent states. This is achieved through a different detection scheme, where the coherent states are unambiguously discriminated. Such an unambiguous state discrimination (USD) is error-free; so for nonorthogonal states, it must include inconclusive measurement results. These will lead to lower efficiencies of the entanglement generation, in particular, when smaller photon numbers are used in order to suppress the decoherence effect through photon losses. The corresponding trade-off between success probability and fidelity means there are ultimate quantum mechanical bounds on the accessible regimes. We will discuss these bounds and propose suboptimal, but practical, linear optical implementations.

The emphasis here is on possible measurement schemes for the initial entanglement generation. Further, we investigate the hybrid entangled atom-light states before the measurements and potential variations of the entanglement distillation and swapping steps. The latter could be performed already on the atom-light level ("hybrid en- tanglement distillation and swapping") rather than solely on the atomic level after the conditional state preparation. We do not consider issues related with the CQED part (for this, see [20]); neither are we concerned about architecture-related issues on how to combine the entanglement purification and swapping steps in an optimal way (for this, see [23]). Such considerations will be needed for comparing the overall efficiencies between the hybrid approach and the single-photon-based schemes.

The plan of the paper is as follows. First, in Sec. II we will examine the hybrid entangled states between one atomic spin and an optical mode (Sec. IIA), the entangled states of two spins and an optical mode (Sec. IIB), and the measurements for conditional entangled-state preparation (Sec. IIC). Secondly, in Sec. III, we will discuss the notions of hybrid entanglement distillation and swapping and their potential realizations.

\section{ENTANGLEMENT GENERATION}

In the hybrid quantum repeater, the mechanism for entanglement distribution is based on dispersive light-matter interactions, obtainable from the JaynesCummings interaction Hamiltonian $\hbar g\left(\hat{\sigma}^{-} \hat{a}^{\dagger}+\hat{\sigma}^{+} \hat{a}\right)$ in

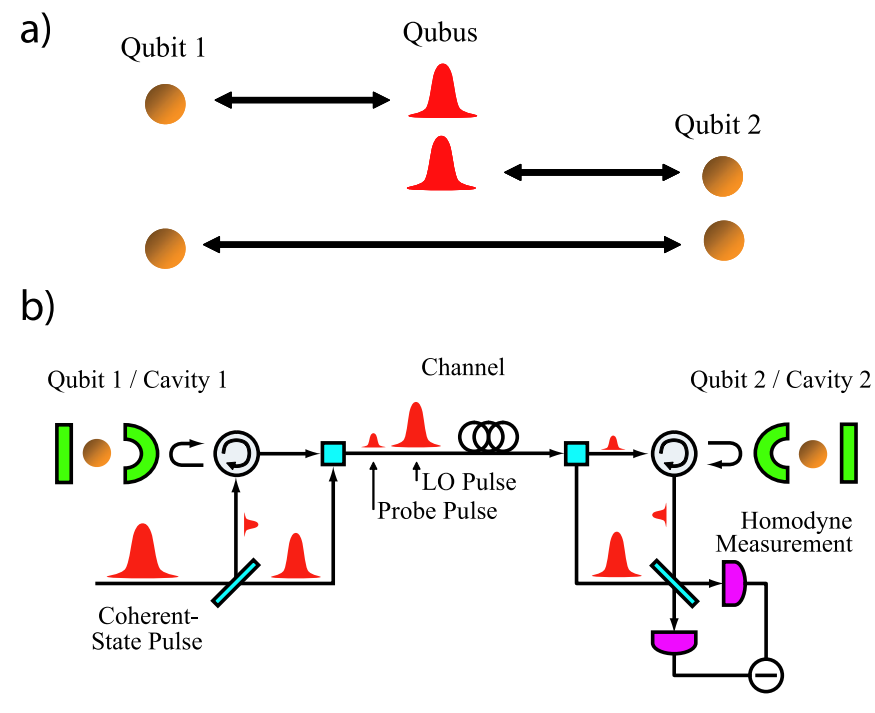

FIG. 1: a) Three steps for the generation of spin-entanglement between two qubits at neighboring repeater stations: the first interaction results in an entangled state between the atomic qubit 1 and the optical qubus; after transmission, the qubus interacts with the atomic qubit 2, leading to a tripartite entangled state between the two qubits and the qubus; finally, a measurement on the qubus mode conditionally creates the two-qubit entanglement. b) Example of a possible measurement scheme for discriminating between the conditionally phase-rotated coherent probe beams in the hybrid quantum repeater; the LO pulse is a sufficiently strong local oscillator used for homodyne detection [19]. 
the limit of large detuning [24],

$$
\hat{H}_{\mathrm{int}}=\hbar \chi \hat{\sigma}_{z} \hat{a}^{\dagger} \hat{a} .
$$

Here, $\hat{a}\left(\hat{a}^{\dagger}\right)$ is the annihilation (creation) operator of the electromagnetic field mode and $\hat{\sigma}_{z}=|0\rangle\langle 0|-| 1\rangle\langle 1|$ is the corresponding qubit Pauli operator for a two-level atom. The parameter $\chi=g^{2} / \Delta$ describes the strength of the atom-light coupling; $2 g$ is the vacuum Rabi splitting for the dipole transition and $\Delta$ is the detuning between the dipole transition and the light field. The Hamiltonian in Eq. (1) leads to a conditional phase-rotation of the field mode,

$$
\hat{U}_{\mathrm{int}}=\exp \left(i \theta \hat{\sigma}_{z} \hat{a}^{\dagger} \hat{a}\right) .
$$

Here, $\chi t \equiv \theta$ is an effective interaction time. The only requirement for a dispersive interaction resulting in a highfidelity conditional rotation is a sufficiently large cooperativity parameter in a weak or intermediate coupling regime; strong coupling is not needed [20]. For simplicity, let us now write the effect of a controlled rotation on a coherent state and a qubit superposition state (corresponding to Eq. (2) up to an uncontrolled phase rotation) as

$$
\hat{U}_{\text {int }}[(|0\rangle+|1\rangle)|\alpha\rangle] / \sqrt{2}=\left(|0\rangle|\alpha\rangle+|1\rangle\left|\alpha e^{i \theta}\right\rangle\right) / \sqrt{2} .
$$

In the following, we will investigate the entangled states that emerge from this interaction. According to the initial entanglement distribution procedure for the hybrid quantum repeater, as a first step, one atomic qubit interacts with the optical qubus mode (see Fig. 11), resulting in a "hybrid entangled state" between qubit 1 and the qubus, as described by Eq. (3). During the transmission of the qubus through the (lossy) channel, the hybrid entangled state is subject to decoherence and becomes mixed. Then the qubus interacts with qubit 2; at this stage, the two qubits and the qubus are in a tripartite (mixed) entangled state. Finally, a measurement on the qubus mode conditionally prepares a two-qubit (mixed) entangled state. An example of a possible measurement scheme to (approximately) achieve this final step is through homodyne detection (see Fig. 1b). Let us now closely examine the elements of this protocol with respect to possible improvements.

\section{A. Qubit-qubus entanglement}

Let us assume the qubus mode is entangled with qubit 1 after the first interaction, as expressed by Eq. (3)). Photon losses in the qubus channel are now described via a simple beam splitter which reflects, on average, $1-\eta$ photons into an environment mode, initially in the vacuum state $|0\rangle_{\mathrm{E}}$,

$$
\begin{aligned}
& |0\rangle_{\mathrm{A}}|\sqrt{\eta} \alpha\rangle_{\mathrm{B}}|\sqrt{1-\eta} \alpha\rangle_{\mathrm{E}} / \sqrt{2} \\
& \quad+|1\rangle_{\mathrm{A}}\left|\sqrt{\eta} \alpha e^{i \theta}\right\rangle_{\mathrm{B}}\left|\sqrt{1-\eta} \alpha e^{i \theta}\right\rangle_{\mathrm{E}} / \sqrt{2}
\end{aligned}
$$

The subscripts "A" and "B" denote the state of the atomic qubit and the qubus mode, respectively. We may now rewrite each of the two pairs of pure, nonorthogonal states of the qubus mode and the loss mode in an orthogonal, two-dimensional basis, $\{|u\rangle,|v\rangle\}$,

$$
\begin{aligned}
|\sqrt{\eta} \alpha\rangle_{\mathrm{B}} & =\mu_{\mathrm{B}}|u\rangle_{\mathrm{B}}+\nu_{\mathrm{B}}|v\rangle_{\mathrm{B}}, \\
\left|\sqrt{\eta} \alpha e^{i \theta}\right\rangle_{\mathrm{B}} & =\left(\mu_{\mathrm{B}}|u\rangle_{\mathrm{B}}-\nu_{\mathrm{B}}|v\rangle_{\mathrm{B}}\right) e^{i \eta \xi}, \\
|\sqrt{1-\eta} \alpha\rangle_{\mathrm{E}} & =\mu_{\mathrm{E}}|u\rangle_{\mathrm{E}}+\nu_{\mathrm{E}}|v\rangle_{\mathrm{E}}, \\
\left|\sqrt{1-\eta} \alpha e^{i \theta}\right\rangle_{\mathrm{E}} & =\left(\mu_{\mathrm{E}}|u\rangle_{\mathrm{E}}-\nu_{\mathrm{E}}|v\rangle_{\mathrm{E}}\right) e^{i(1-\eta) \xi},
\end{aligned}
$$

where $\nu_{\mathrm{B}}=\sqrt{1-\mu_{\mathrm{B}}^{2}}$ and $\nu_{\mathrm{E}}=\sqrt{1-\mu_{\mathrm{E}}^{2}}$ with

$$
\begin{aligned}
& \mu_{\mathrm{B}}=\left[1+e^{-\eta \alpha^{2}(1-\cos \theta)}\right]^{1 / 2} / \sqrt{2}, \\
& \mu_{\mathrm{E}}=\left[1+e^{-(1-\eta) \alpha^{2}(1-\cos \theta)}\right]^{1 / 2} / \sqrt{2},
\end{aligned}
$$

and $\xi \equiv \alpha^{2} \sin \theta$. Here and in the following, we assume $\alpha$ to be real. Now tracing over the loss mode and using the "A" basis, $\left\{\left(|0\rangle_{\mathrm{A}} \pm e^{i \xi}|1\rangle_{\mathrm{A}}\right) / \sqrt{2}\right\}$, as new computational basis, we can express the "two-qubit" density matrix of "A" and "B" in a very compact way as

$\mu_{\mathrm{E}}^{2}\left|\Phi^{+}\left(\mu_{\mathrm{B}}\right)\right\rangle\left\langle\Phi^{+}\left(\mu_{\mathrm{B}}\right)\left|+\left(1-\mu_{\mathrm{E}}^{2}\right)\right| \Psi^{+}\left(\mu_{\mathrm{B}}\right)\right\rangle\left\langle\Psi^{+}\left(\mu_{\mathrm{B}}\right)\right|$.

The resulting density matrix is a mixture of two nonmaximally entangled states,

$$
\begin{aligned}
& \left|\Phi^{+}\left(\mu_{\mathrm{B}}\right)\right\rangle=\mu_{\mathrm{B}}|0\rangle_{\mathrm{A}}|u\rangle_{\mathrm{B}}+\sqrt{1-\mu_{\mathrm{B}}^{2}}|1\rangle_{\mathrm{A}}|v\rangle_{\mathrm{B}}, \\
& \left|\Psi^{+}\left(\mu_{\mathrm{B}}\right)\right\rangle=\mu_{\mathrm{B}}|1\rangle_{\mathrm{A}}|u\rangle_{\mathrm{B}}+\sqrt{1-\mu_{\mathrm{B}}^{2}}|0\rangle_{\mathrm{A}}|v\rangle_{\mathrm{B}} .
\end{aligned}
$$

The phase-rotated coherent states are now contained in the orthogonal basis of the corresponding qubus-mode subspace,

$$
\begin{aligned}
|u\rangle_{\mathrm{B}} & =\frac{1}{2 \mu_{\mathrm{B}}}\left(|\sqrt{\eta} \alpha\rangle_{\mathrm{B}}+e^{-i \eta \xi}\left|\sqrt{\eta} \alpha e^{i \theta}\right\rangle_{\mathrm{B}}\right) \\
|v\rangle_{\mathrm{B}} & =\frac{1}{2 \sqrt{1-\mu_{\mathrm{B}}^{2}}}\left(|\sqrt{\eta} \alpha\rangle_{\mathrm{B}}-e^{-i \eta \xi}\left|\sqrt{\eta} \alpha e^{i \theta}\right\rangle_{\mathrm{B}}\right) .
\end{aligned}
$$

In the form of Eq. (8), one can easily observe the tradeoff of the presence of entanglement for different photon numbers $\alpha^{2}$, assuming imperfect transmission, $\eta<1$, and reasonable phase shifts, $\theta \sim 10^{-2}, 10^{-3}$. Choosing $\alpha$ small means the density matrix in Eq. (8) approaches a pure state, according to Eq. (7); however, this pure state is nearly unentangled for too small $\alpha$, according to Eqs. (6), (9). Conversely, for large $\alpha$, we have $\mu_{\mathrm{E}}^{2} \rightarrow 1 / 2$, leading to an almost equal mixture of the states of Eqs. (9),(10) in Eq. (8); however, this time, the individual states of Eqs. (9),(10) are nearly maximally entangled Bell states. In other words, the quality of the entanglement is affected either by the decoherence effect of the channel (for large $\alpha$, when many photons transfer which-path information into the environment) or by the nonmaximal entanglement of the pure states 


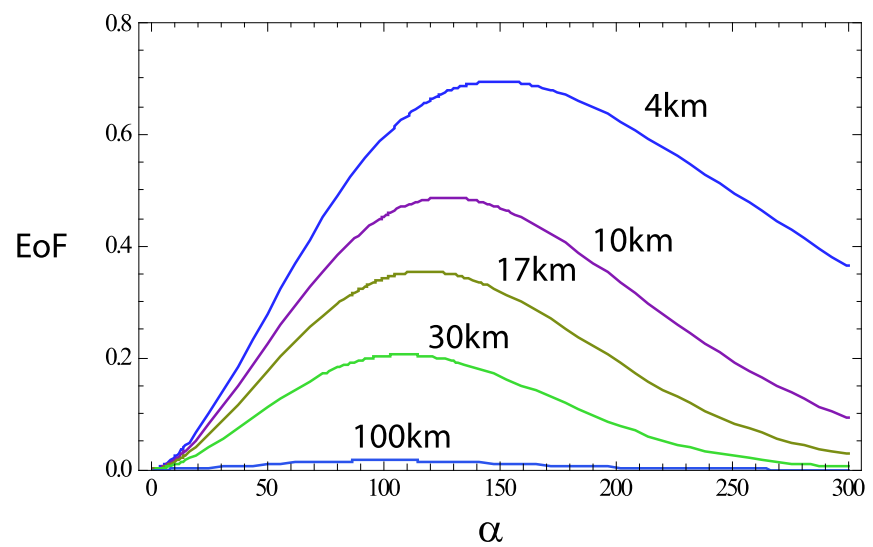

FIG. 2: The entanglement of formation of the qubit1-qubus states as a function of the qubus amplitude $\alpha$ (square root of qubus photon number) for different channel transmissions, i.e., different distances of qubus propagation; photon loss is assumed to be $0.18 \mathrm{~dB}$ per $\mathrm{km}$. The phase $\theta$ is always 0.01 .

in Eqs. (9), (10) (for small $\alpha$, when the phase-rotated coherent states are nearly indistinguishable and the initial atom-light entanglement is weak).

Another interesting feature of the state in Eq. (8) is that we may consider a purification of some copies of it through local operations on the qubits and the qubus modes, hence distilling a higher degree of entanglement into a smaller number of copies. This "prepurification" (prior to the qubus interaction with qubit 2) or "hybrid entanglement distillation" will be discussed in Sec. III A

As the density matrix in Eq. (8) effectively describes a two-qubit state, we can evaluate its entanglement of formation using the concurrence 25]. Figure 2 shows the entanglement of formation as a function of the qubus amplitude $\alpha$ (square root of qubus photon number) for different channel transmissions. Note that this is the maximum initial entanglement (prior to any entanglement distillation procedures) available in the repeater protocol. All the remaining steps of the initial entanglement generation, including the interaction between qubus and qubit 2 and the measurement of the qubus mode, are local; hence they can only reduce the amount of entanglement. The optimal value of the product $\alpha \theta$, maximizing the entanglement, is always of the order of $\alpha \theta \sim 1$. This is similar to the result obtained for the two-qubit singlet fidelity after the interaction with qubit 2 and homodyne measurement of the qubus mode, reflecting the optimal trade-off between distinguishability and decoherence [19]. Let us now consider the interaction of the qubus mode with qubit 2 and look at the resulting tripartite mixed entangled state.

\section{B. Qubit-qubus-qubit entanglement}

In the hybrid quantum repeater protocol, the entangled state of Eq. (8) is subject to a second interaction, this time between the qubus mode and qubit 2 (which initially is in an equal superposition state). This interaction results in a controlled rotation of the qubus by an angle of $-\theta$, as described by Eq. (3) with $\theta \rightarrow-\theta$. The controlled rotation transforms the two orthogonal qubus basis states of Eq. (11) together with the qubit state as

$$
\begin{aligned}
|u\rangle_{\mathrm{B}} \otimes\left(|0\rangle_{\mathrm{C}}+|1\rangle_{\mathrm{C}}\right) / \sqrt{2} & \rightarrow \\
\frac{1}{2 \sqrt{2}} & {\left[|\sqrt{\eta} \alpha\rangle_{\mathrm{B}}\left(|0\rangle_{\mathrm{C}}+e^{-i \eta \xi}|1\rangle_{\mathrm{C}}\right)\right.} \\
& +e^{-i \eta \xi}\left|\sqrt{\eta} \alpha e^{i \theta}\right\rangle_{\mathrm{B}}|0\rangle_{\mathrm{C}} \\
& \left.+\left|\sqrt{\eta} \alpha e^{-i \theta}\right\rangle_{\mathrm{B}}|1\rangle_{\mathrm{C}}\right] / \mu_{\mathrm{B}},
\end{aligned}
$$

and, similarly,

$$
\begin{aligned}
|v\rangle_{\mathrm{B}} \otimes\left(|0\rangle_{\mathrm{C}}+|1\rangle_{\mathrm{C}}\right) / \sqrt{2} & \rightarrow \\
\frac{1}{2 \sqrt{2}} & {\left[|\sqrt{\eta} \alpha\rangle_{\mathrm{B}}\left(|0\rangle_{\mathrm{C}}-e^{-i \eta \xi}|1\rangle_{\mathrm{C}}\right)\right.} \\
& -e^{-i \eta \xi}\left|\sqrt{\eta} \alpha e^{i \theta}\right\rangle_{\mathrm{B}}|0\rangle_{\mathrm{C}} \\
& \left.+\left|\sqrt{\eta} \alpha e^{-i \theta}\right\rangle_{\mathrm{B}}|1\rangle_{\mathrm{C}}\right] / \sqrt{1-\mu_{\mathrm{B}}^{2}}
\end{aligned}
$$

Applying these transformations to the density matrix in Eq. (8), a local Hadamard gate to the spin system "A", and a local rotation $e^{i \eta \xi\left(1-\hat{\sigma}_{z}\right) / 2}$ upon system "C", leads to the following tripartite density operator,

$$
\mu_{\mathrm{E}}^{2}\left|\Phi^{+}\right\rangle\left\langle\Phi^{+}\left|+\left(1-\mu_{\mathrm{E}}^{2}\right)\right| \Phi^{-}\right\rangle\left\langle\Phi^{-}\right|,
$$

where

$$
\begin{aligned}
\left|\Phi^{+}\right\rangle=\frac{1}{\sqrt{2}}|\sqrt{\eta} \alpha\rangle_{\mathrm{B}}\left|\phi^{+}\right\rangle_{\mathrm{AC}} & +\frac{1}{2} e^{-i \eta \xi}\left|\sqrt{\eta} \alpha e^{i \theta}\right\rangle_{\mathrm{B}}|10\rangle_{\mathrm{AC}} \\
& +\frac{1}{2} e^{i \eta \xi}\left|\sqrt{\eta} \alpha e^{-i \theta}\right\rangle_{\mathrm{B}}|01\rangle_{\mathrm{AC}}, \\
\left|\Phi^{-}\right\rangle=\frac{1}{\sqrt{2}}|\sqrt{\eta} \alpha\rangle_{\mathrm{B}}\left|\phi^{-}\right\rangle_{\mathrm{AC}} & -\frac{1}{2} e^{-i \eta \xi}\left|\sqrt{\eta} \alpha e^{i \theta}\right\rangle_{\mathrm{B}}|10\rangle_{\mathrm{AC}} \\
+ & \frac{1}{2} e^{i \eta \xi}\left|\sqrt{\eta} \alpha e^{-i \theta}\right\rangle_{\mathrm{B}}|01\rangle_{\mathrm{AC}},
\end{aligned}
$$

with the maximally entangled Bell states $\left|\phi^{ \pm}\right\rangle=(|00\rangle \pm$ $|11\rangle) / \sqrt{2}$. The state in Eq. (14) with Eqs. (15) again illustrates the two competing sources of errors in the entanglement generation step. Bit-flip errors are caused by the indistinguishability of the phase-rotated coherent states (if homodyne detection is used for state discrimination); these can be reduced via sufficiently large photon numbers. Phase-flip errors occur for any imperfect transmission; this decoherence effect is suppressed for smaller photon numbers.

In the following, we will consider unambiguous state discrimination (USD) 26, 27, 28] of the corresponding phase-rotated coherent states in Eq. (14). This enables us to completely eliminate bit-flip errors, at the expense of a reduced efficiency coming from inconclusive measurement results. The relevant, quantum mechanical USD problem provides ultimate performance bounds. These shall be approached using practical linear-optics solutions for the required generalized measurements. 


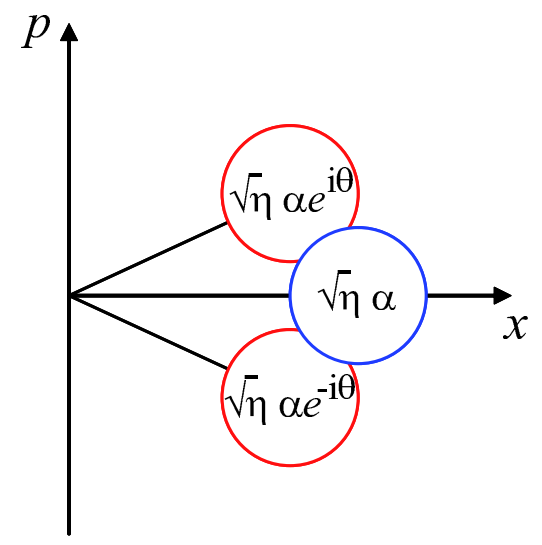

FIG. 3: Phase-rotated coherent states to be discriminated for entangled-state preparation; the unrotated state belongs to the preferred qubit subspace (even parity); the two rotated states are correlated with the odd subspace of the two qubits.

\section{Conditional state preparation}

As illustrated in Fig. 1a, after the interaction of the qubus with qubit 2 , the final step is to prepare conditionally an entangled two-qubit state through measurements on the qubus mode including postselection. The postselection procedure may either filter out approximate, mixed entangled two-qubit states, still containing some errors from the finite overlap of the phase-rotated coherent states [19]; or it may, at a lower succcess rate, perfectly rule out those contributions belonging to the "wrong" coherent states (see Fig. 3) and project onto an entangled two-qubit state whose imperfection originates solely from the losses in the communication channel. The latter scenario can be achieved via a scheme based on USD measurements, providing the ultimate, distance-dependent limits on the quality of the initially generated entangled states. At the same time, it yields an alternative approach to feasible implementations of the entanglement generation step. Let us first briefly recall the homodyne-based entanglement generation scheme.

\section{Homodyne-based state preparation}

A very efficient and practical way to discriminate the phase-rotated coherent states in Fig. 3] is through homodyne detection [19]. While an $x$ measurement could, in principle, project onto both the even and the odd qubit subspace, a $p$ measurement only leads to an entangled state in the even subspace and those results consistent with either the $|10\rangle$ or the $|01\rangle$ state must be discarded. Nonetheless, for the quantum repeater protocol, the $p$ measurement is preferred to the $x$ measurement, as the errors in the former scale as $\alpha \theta$ and those of the latter as $\alpha \theta^{2}$, resulting in fast decoherence for small $\theta$ and $\alpha$ sufficiently large. Let us now discuss the ultimate bounds on the performance of the entanglement generation step using an error-free, USD-based measurement scheme.

\section{Ultimate bounds}

In order to derive some bounds on the attainable entangled-state fidelities at a certain rate for a given distance, let us consider the binary USD problem of discriminating the state $|\sqrt{\eta} \alpha\rangle$ versus the set of states $\left\{\left|\sqrt{\eta} \alpha e^{i \theta}\right\rangle,\left|\sqrt{\eta} \alpha e^{-i \theta}\right\rangle\right\}$. We may rephrase this problem as the USD of the two density operators

$$
\begin{aligned}
& \hat{\rho}_{1}=|\sqrt{\eta} \alpha\rangle\langle\sqrt{\eta} \alpha|, \\
& \hat{\rho}_{2}=\frac{1}{2}\left(\left|\sqrt{\eta} \alpha e^{i \theta}\right\rangle\left\langle\sqrt{\eta} \alpha e^{i \theta}|+| \sqrt{\eta} \alpha e^{-i \theta}\right\rangle\left\langle\sqrt{\eta} \alpha e^{-i \theta}\right|\right) .
\end{aligned}
$$

Any measurement scheme which is intended to filter out unambiguously an entangled Bell state from the two individual states in Eqs. (15) of the mixture in Eq. (14) must be a solution to the above USD problem; thus, the best possible entanglement generation scheme cannot outperform the optimal USD scheme. However, note that the converse is not true. A generalized measurement, even for optimal USD, does not necessarily result in an entangled state. In particular, unambiguously identifying the state $\hat{\rho}_{2}$ in Eq. (15) may also mean that the sign of the phase rotation is determined. In this case, the two qubits end up in a separable state, according to Eq. (14) with Eqs. (15). In fact, in order to coherently project onto the odd qubit subspace (as it could be done erroneously via $x$ homodyne detection, see Fig. (3) through USD, the measurement scheme becomes less practical involving photon number resolving detectors [29].

The USD of mixed quantum states is a much more subtle issue than that of pure states [30, 31, 32, 33]. Nonetheless, for equal a priori probabilities (which is the

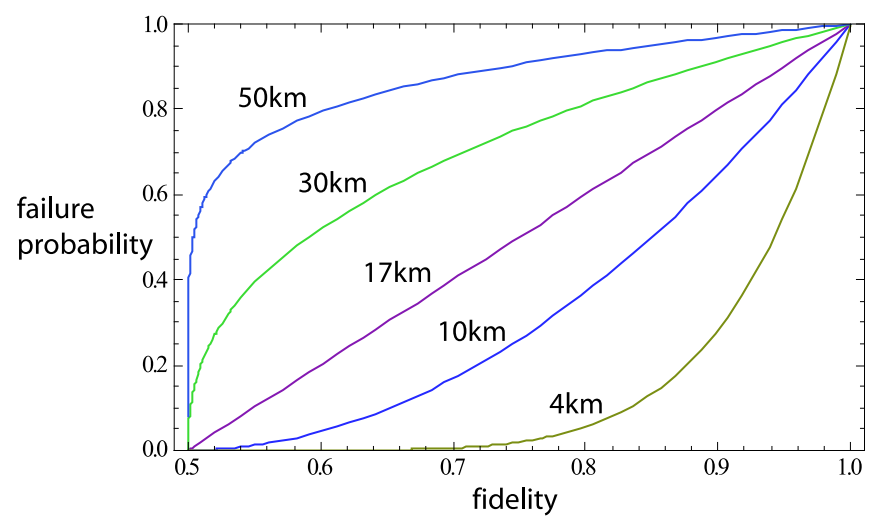

FIG. 4: The optimal failure probability for unambiguous state discrimination of the two density operators in Eq. (15) as a function of the fidelity of the desired entangled two-qubit state in Eq. (14). The regions below each curve are quantum mechanically inaccessible. Note that for a transmission $\eta=$ $1 / 2(17 \mathrm{~km})$, the functional dependence is linear. 
case we are interested in), the failure probability (the probability for obtaining an inconclusive measurement outcome) is bounded from below by the square root of the fidelity of the two density operators [32]. For the USD problem of Eq. (15), this means

$$
P_{?} \geq \sqrt{\left\langle\sqrt{\eta} \alpha\left|\hat{\rho}_{2}\right| \sqrt{\eta} \alpha\right\rangle}
$$

Using Eq. (15), this leads to the optimal (minimal) failure probability

$$
P_{?}^{\mathrm{opt}}=e^{-\eta \alpha^{2}(1-\cos \theta)}
$$

This bound can be inserted into the fidelity (this time for the qubit states) $\mu_{\mathrm{E}}^{2} \equiv F$ of the desired $\left|\Phi^{+}\right\rangle$state in the mixture of Eq. (14). Using Eq. (7), we obtain

$$
P_{?}^{\mathrm{opt}}(F)=(2 F-1)^{\eta /(1-\eta)}
$$

The optimal failure probability as a function of the fidelity is shown in Fig. 4 for different distances.

The larger the distances, the larger the failure probabilities become at a given fidelity. Reasonably high fidelities are only achievable at the expense of small success probabilities. However, the bound for the USD problem does allow for fidelities much greater than $1 / 2$ at distances of $50 \mathrm{~km}$ and more. We will now investigate whether there are practical implementations of the corresponding USD measurement which approach the quantum mechanical bounds and hence are no longer limited by distances of $20 \mathrm{~km}$ and below.

\section{Unambiguous state preparation}

Apart from an initial entanglement generation over potentially larger distances, there are other advantages of using USD for the conditional entangled-state preparation. In particular, the resulting imperfect entangled states will be mixtures of just two Bell states (rank two mixtures), in the form of Eq. (14) after ruling out the odd parity terms in Eqs. (15). For some copies of this type of mixed-entangled states, entanglement distillation is more efficient 34] and the so-called entanglement pumping is no longer bounded by some fidelity threshold below unity (as for higher rank Bell-diagonal mixtures) [4]; entanglement pumping means that spatial resources in the repeater protocol can be turned into temporal resources by distilling always the same entangled pair with the help of freshly prepared elementary pairs.

A scheme for unambiguously discriminating the phaserotated coherent states in Fig. 3 and hence realizing USD of the density matrices in Eq. (15), based upon linear optics and photon detection, is shown in Fig. 5. The qubus mode is sent through a linear three-port device, together with two ancilla vacuum modes, and subsequently, the three output modes are displaced in phase space before being detected. The three-port device acts upon a coherent state $|\beta\rangle$ as

$$
|\beta, 0,0\rangle \rightarrow\left|\lambda \beta, \lambda \beta, \sqrt{1-2 \lambda^{2}} \beta\right\rangle
$$

choosing $\lambda$ real. The subsequent phase-space displacements are

$$
\begin{gathered}
\hat{D}\left(-\lambda \sqrt{\eta} \alpha e^{i \theta}\right) \otimes \hat{D}\left(-\lambda \sqrt{\eta} \alpha e^{-i \theta}\right) \\
\otimes \hat{D}\left(-\sqrt{1-2 \lambda^{2}} \sqrt{\eta} \alpha\right)
\end{gathered}
$$

Via the three-port device and the displacements, the three different qubus input states to be discriminated are transformed as

$$
\begin{aligned}
& |\sqrt{\eta} \alpha, 0,0\rangle \rightarrow\left|\lambda \sqrt{\eta} \alpha\left(1-e^{i \theta}\right), \lambda \sqrt{\eta} \alpha\left(1-e^{-i \theta}\right), 0\right\rangle, \\
& \left|\sqrt{\eta} \alpha e^{i \theta}, 0,0\right\rangle \rightarrow \\
& \left.\quad \mid 0, \lambda \sqrt{\eta} \alpha 2 i \sin \theta), \sqrt{1-2 \lambda^{2}} \sqrt{\eta} \alpha\left(e^{i \theta}-1\right)\right\rangle \\
& \left|\sqrt{\eta} \alpha e^{-i \theta}, 0,0\right\rangle \rightarrow \\
& \left.\quad \mid-\lambda \sqrt{\eta} \alpha 2 i \sin \theta), 0, \sqrt{1-2 \lambda^{2}} \sqrt{\eta} \alpha\left(e^{-i \theta}-1\right)\right\rangle .
\end{aligned}
$$

There are now six out of eight possible detection patterns, considering detectors which do not resolve photon numbers (going either "click" or "no click"). These patterns unambiguously identify the corresponding coherent states of the input,

$$
\begin{aligned}
& \mid \text { click, click, no click }\rangle \rightarrow|\sqrt{\eta} \alpha\rangle \\
& \mid \text { no click, no click, click }\rangle \rightarrow\left|\sqrt{\eta} \alpha e^{ \pm i \theta}\right\rangle, \\
& \mid \text { no click, click, click }\rangle \rightarrow\left|\sqrt{\eta} \alpha e^{i \theta}\right\rangle \\
& \mid \text { click, no click, click }\rangle \rightarrow\left|\sqrt{\eta} \alpha e^{-i \theta}\right\rangle, \\
& \mid \text { no click, click, no click }\rangle \rightarrow|\sqrt{\eta} \alpha\rangle \text { or }\left|\sqrt{\eta} \alpha e^{i \theta}\right\rangle, \\
& \mid \text { click, no click, no click }\rangle \rightarrow|\sqrt{\eta} \alpha\rangle \text { or }\left|\sqrt{\eta} \alpha e^{-i \theta}\right\rangle .
\end{aligned}
$$

The pattern |noclick, noclick, noclick $\rangle$ is inconclusive (corresponding to the vacuum contributions from all three modes), whereas the pattern $\mid$ click, click, click $\rangle$ does not occur at all.

Among the remaining six detection patterns, there are two patterns which are inconclusive, in

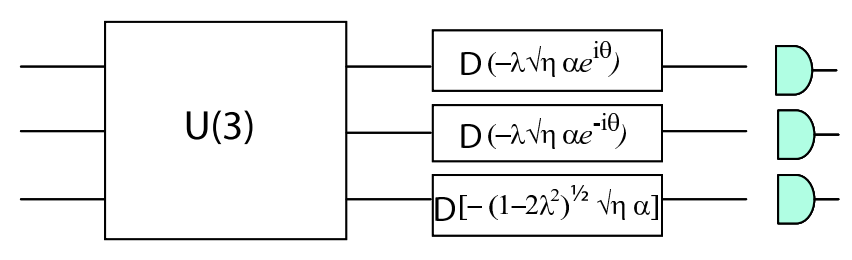

FIG. 5: Unambiguous state discrimination of a zero-phase coherent state from two phase-rotated coherent states via linear optics, phase-space displacements, and photon detection. 
addition to the vacuum-based, inconclusive pattern |noclick, noclick, no click $\rangle$. The extra inconclusive patterns are |noclick, click, noclick $\rangle$ and $\mid$ click, noclick, noclick $\rangle$ (the last two patterns of Eq. (22)). As they rule out only $\left|\sqrt{\eta} \alpha e^{-i \theta}\right\rangle$ and $\left|\sqrt{\eta} \alpha e^{i \theta}\right\rangle$, respectively, they are conclusive results neither for the USD problem nor for the entanglement generation in the repeater protocol. However, the conditional states that emerge after the detection of these patterns (i.e., after obtaining just one click in either the first or the second mode) are, in principle, still usable for both USD and entanglement generation. Here we will not consider such conditional dynamics.

Focusing on the remaining four patterns, we observe the following. Any one of the first four patterns of Eq. (22) conclusively identifies the quantum state in the USD problem of Eq. (15). Among these four, only the first pattern, $\mid$ click, click, no click $\rangle$, identifies the state $|\sqrt{\eta} \alpha\rangle$. The other three patterns are only consistent with the state $\hat{\rho}_{2}$ in Eq. (15), ruling out $\hat{\rho}_{1}$.

If we now look at the entanglement generation step of the repeater protocol, then even the patterns |no click, click, click $\rangle$ and |click, no click, click $\rangle$ must count as failure, because conclusively identifying either the state $\left|\sqrt{\eta} \alpha e^{i \theta}\right\rangle$ or the state $\left|\sqrt{\eta} \alpha e^{-i \theta}\right\rangle$ means that the two atomic spins will end up in a separable state, according to Eq. (14) with Eqs. (15).

Eventually, only the two patterns |click, click, no click $\rangle$ and |noclick, noclick, click $\rangle$ are useful for the entanglement generation. The former one, conclusively identifying the state $|\sqrt{\eta} \alpha\rangle$, projects the two qubits onto the even parity subspace. The latter one, ruling out $|\sqrt{\eta} \alpha\rangle$ and being consistent with both $\left|\sqrt{\eta} \alpha e^{i \theta}\right\rangle$ and $\left|\sqrt{\eta} \alpha e^{-i \theta}\right\rangle$, leads to the odd subspace. However, in this case, just obtaining a click for mode 3 is not enough to project the two qubits onto a maximally entangled state, not even in the ideal case without losses (see Eq. (14)). Such a measurement would result in a superposition of the two "odd" Bell states $\left|\psi^{ \pm}\right\rangle=(|10\rangle \pm|01\rangle) / \sqrt{2}$ with an even number (without the vacuum) and an odd number coherent-state superposition, $\approx|\alpha i \theta\rangle \pm|-\alpha i \theta\rangle$, respectively. Thus, only through detection of the photon number parity, a maximally entangled Bell state of the two qubits can emerge. This could be achieved via photon number resolving detectors [29].

As a result, we obtain a highly practical solution for entanglement generation, based upon two detectors firing at the same time, $\mid$ click, click, no click $\rangle$. We denote the success probability for this event to occur as $P^{\text {even }}$. Similarly, the probability for projecting onto the odd subspace shall be $P^{\text {odd,USD }}$ and $P^{\text {odd,ent }}$, where $P^{\text {odd, USD in- }}$ cludes those patterns which may or may not resolve the two states $\left|\sqrt{\eta} \alpha e^{i \theta}\right\rangle$ and $\left|\sqrt{\eta} \alpha e^{-i \theta}\right\rangle$ and hence are only partly useful for entanglement generation (but still entirely for USD). The probability $P^{\text {odd,ent }}$ only includes the pattern |no click, no click, click $\rangle$, which, using photon number resolving detectors, leads to an entangled state.
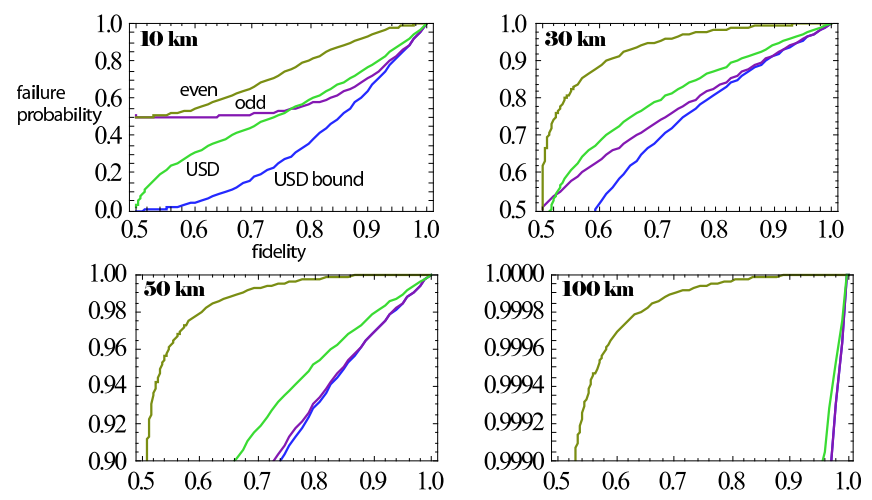

FIG. 6: Failure probabilities as functions of the final two-qubit maximally entangled-state fidelities for different distances. The regions below "USD bound" are quantum mechanically inaccessible. The curves "USD" correspond to those linear-optics implementations in which all conclusive patterns for both even and odd subspaces are combined (choosing a beam splitter parameter $\lambda=0.4)$. The plots for "even" $(\lambda=0.7)$ and "odd" $(\lambda=0.01)$ describe those measurement schemes where only a single detection pattern is used in order to project onto the respective two-qubit subspaces (|click, click, no click $\rangle$ for "even" and |no click, no click, click $\rangle$ for "odd").

For these probabilities, we obtain,

$$
\begin{aligned}
P^{\text {even }} & =\frac{1}{2}\left(1-e^{-\lambda^{2} \eta \alpha^{2} 2(1-\cos \theta)}\right)^{2}, \\
P^{\text {odd, USD }} & =\frac{1}{2}\left(1-e^{-\left(1-2 \lambda^{2}\right) \eta \alpha^{2} 2(1-\cos \theta)}\right), \\
P^{\text {odd,ent }} & =P^{\text {odd,USD }} \times e^{-\lambda^{2} \eta \alpha^{2} 4 \sin ^{2} \theta} .
\end{aligned}
$$

Finally, we use $P^{\text {total,USD }}=P^{\text {even }}+P^{\text {odd,USD }}$ and $P^{\text {total,ent }}=P^{\text {even }}+P^{\text {odd,ent }}$ to describe the corresponding total success probabilities.

Compared to the ultimate bounds derived in Sec. ПC2, we may now consider three different scenarios. First, the most practical scheme for entanglement generation, namely, by unambiguously identifying the state $|\sqrt{\eta} \alpha\rangle$, projecting the two qubits onto a mixture of even-parity entangled Bell states. This scheme works with a probability of $P^{\text {even }}$ (which is always smaller than $1 / 2$ ), and does not require photon number resolving detectors. Secondly, we consider the odd qubit subspace for entanglement generation, resulting in a slightly less practical scheme with a need for photon number resolving detectors; the probability here is $P^{\text {odd,ent }}$. Finally, we add those patterns which resolve the states $\left|\sqrt{\eta} \alpha e^{i \theta}\right\rangle$ and $\left|\sqrt{\eta} \alpha e^{-i \theta}\right\rangle$ to consider the total probability for a reasonably practical USD scheme, $P^{\text {total,USD }}$. This comparison is shown in Fig. 6] where the success probabilities are replaced by failure probabilities, as functions of the fidelity $\mu_{\mathrm{E}}^{2} \equiv F$ in Eq. (14). The linear-optics parameter $\lambda$ can be used to tune between the even and the odd subspaces.

In Fig. 6, again the regions below "USD bound" 
are quantum mechanically inaccessible. The curves "USD" correspond to those linear-optics implementations in which all conclusive patterns for both even and odd subspaces are combined (choosing a beam splitter parameter $\lambda=0.4$ ); the failure probabilities shown correspond to $1-P^{\text {total,USD }}$. The plots for "even" $(\lambda=0.7)$ and "odd" $(\lambda=0.01)$ describe those measurement schemes where only a single detection pattern is used in order to project onto the respective two-qubit subspaces (|click, click, no click $\rangle$ for "even" and $\mid$ no click, no click, click $\rangle$ for "odd"); the failure probabilities shown correspond to $1-P^{\text {even }}$ and $1-P^{\text {odd,ent, }}$ respectively.

Here, "even" is less efficient, but more practical than "odd", as it does not require photon number resolving detectors. Note that for entanglement generation, tuning the beam splitter parameter $\lambda$ in order to project onto both even and odd subspaces at the same time (as for "USD" with $\lambda=0.4$ ) does not lead to better performances. For larger distances, also for the case of "USD", beam splitter tuning no longer helps; either projecting onto the even $(\lambda=0.7)$ or the odd $(\lambda=0.01)$ subspace is optimal in this case as well. Therefore, "USD" performs worse than "odd" for $30 \mathrm{~km}$ and beyond.

Note that the three patterns of Eq. (22) with clicks in every mode except one unambiguously identify each individual state of the set $\left\{|\sqrt{\eta} \alpha\rangle,\left|\sqrt{\eta} \alpha e^{i \theta}\right\rangle,\left|\sqrt{\eta} \alpha e^{-i \theta}\right\rangle\right\}$. In other words, for different $\lambda$, one obtains a family of solutions to the corresponding trinary USD problem. As the three coherent states here are not symmetrically distributed $\left(\exp \left(i \theta \hat{a}^{\dagger} \hat{a}\right)\left|\sqrt{\eta} \alpha e^{i \theta}\right\rangle \neq\left|\sqrt{\eta} \alpha e^{-i \theta}\right\rangle\right.$ for $\theta \neq 2 \pi / 3$ ), the success probability of the quantum mechanically optimal USD is actually not known. For realizing optimal USD of $N$ symmetrically distributed coherent states, protocols have been proposed by van Enk [35], similar to the scheme here, approximately implementing the optimal $N$-state USD.

\section{ENTANGLEMENT PURIFICATION AND SWAPPING}

In the original hybrid quantum repeater proposal [19], the first step is to distribute two-qubit entanglement between nearest-neighbor stations at a high rate, but with rather modest fidelities. In order to achieve high-fidelity quantum communication over the entire repeater channel, the imperfectly entangled qubit states must be purified; the resulting high-fidelity pairs can be used to connect the segments of the channel through entanglement swapping. Further rounds of entanglement purification and swapping will eventually produce a high-fidelity entangled pair between the remote ends of the channel [3, 4].

Instead of using standard entanglement distillation and swapping procedures on the level of the qubits [4, 5, 6, 7, 9], we may also consider a purification of the imperfectly entangled, light-matter hybrid pairs, as

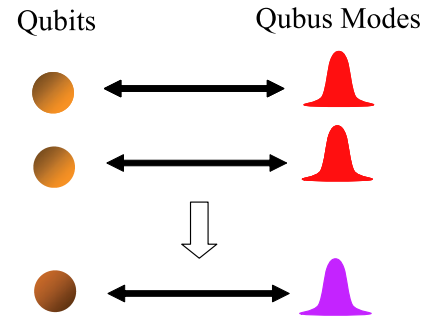

FIG. 7: Purifying two copies of a light-matter hybrid entangled pair into one copy with higher fidelity (purity) through local operations on the qubits and the light modes.

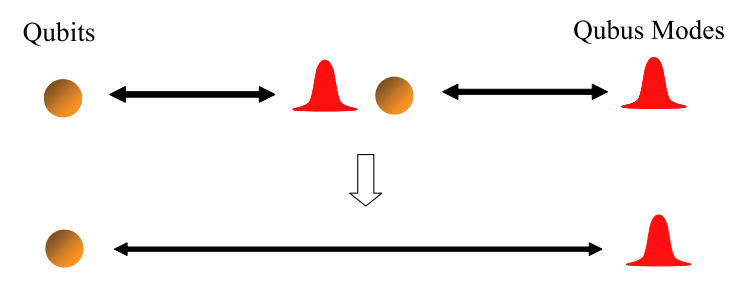

FIG. 8: Hybrid entanglement swapping through unambiguous Bell-state measurements on the joint systems of qubit and light mode.

described by Eq. (8). In this case, local operations would partly act upon the qubits and partly on the light field (see Fig.7). This potentially reduces the number of qubit resources and, moreover, only high-fidelity entanglement would be transferred from the light modes to the qubits. Similarly, we could employ a hybrid version of entanglement swapping, where the Bell-state measurements are performed on the light-matter hybrid systems (Fig. 8).

\section{A. Hybrid entanglement distillation}

Entanglement purification of optical, non-hybrid, entangled coherent states has been considered in Ref. [36]. Provided the initial copies of mixed entangled states are of a specific form (rank two mixtures of a certain pair of entangled coherent, quasi-Bell states), simple linear optics and photon detectors suffice to enhance the fidelity of the entangled states. However, for realistic, dissipative environments, the decohered states would not end up in the desired form; additional local Hadamardtype gates (transforming Gaussian coherent states into non-Gaussian superpositions of coherent states) would be needed in order to accomplish the entanglement purification protocol.

The situation turns out to be similar for the present hybrid protocol. As can be inferred from Eq. (8) with Eqs. (9),(10), the local Hadamard gates in an entanglement purification scheme must act upon the qubit states of system "A" and the coherent-state superposition basis states of the qubus system "B", Eq. (11). Even though there are recent proposals to achieve such logical gates for 
coherent-state superpositions ("coherent-state quantum computing" 37, 38]), using off-line prepared coherentstate superpositions, the need for extra non-Gaussian, optical resources (or, possibly, extra CQED-based, optical resources) may be just as expensive as using additional cavity-based qubit resources. Therefore we conclude that hybrid entanglement distillation does not appear to be a more practical alternative to the standard distillation procedures solely on the level of the two-qubit states.

\section{B. Hybrid entanglement swapping}

In a hybrid version of entanglement swapping, the Bellstate measurements are performed on the light-matter hybrid systems (Fig. 8). For this hybrid Bell measurement, we can just use the same CQED interactions as for the initial entanglement distribution, described by Eq. (3); that interaction provides the entangling gate needed for a projection onto the hybrid "Bell basis". A subsequent Hadamard gate can be applied to the qubit system before measuring both the qubit and the optical qubus in the "computational basis".

More precisely, the following "Bell states" are to be discriminated,

$$
\begin{gathered}
\left(|0\rangle|\alpha\rangle \pm|1\rangle\left|\alpha e^{i \theta}\right\rangle\right) / \sqrt{2} \\
\left(|0\rangle\left|\alpha e^{i \theta}\right\rangle \pm|1\rangle|\alpha\rangle\right) / \sqrt{2}
\end{gathered}
$$

In order to distinguish these states, an interaction gate similar to Eq. (3) is applied, where $|0\rangle|\alpha\rangle \rightarrow|0\rangle|\alpha\rangle$ and $|1\rangle|\alpha\rangle \rightarrow|1\rangle\left|\alpha e^{-i \theta}\right\rangle$. The first pair of Bell states in Eq. (25) is transformed into $(|0\rangle \pm|1\rangle)|\alpha\rangle / \sqrt{2}$; in this case, measuring in the Hadamard-rotated qubit basis reveals the phase of the initial Bell state. In order to additionally identify the second pair in Eq. (25), which will be transformed into $\left(|0\rangle\left|\alpha e^{i \theta}\right\rangle \pm|1\rangle\left|\alpha e^{-i \theta}\right\rangle\right) / \sqrt{2}$, apart from the qubit Hadamard gate and qubit detection, a measurement on the optical qubus mode must discriminate the unrotated coherent state from the two rotated ones in phase space (see Fig. 3).

For a nearly complete Bell measurement (approximately identifying any one of the four hybrid Bell states), the two rotated coherent states must not be distinguished by the measurement. This could be achieved via $x$ homodyne detection. However, as discussed previously, the distinguishability in phase space scales badly with distance along the $x$ axis. Therefore, for a partial Bell measurement identifying only half of the Bell states, either $p$ homodyne detection can be used, or, alternatively, the USD-based scheme for unambiguously detecting the unrotated coherent state, as introduced in the preceding sections. In either case, $p$ homodyne or USD measurement, the efficiency of the Bell measurement would be limited by $1 / 2$.

Now using the hybrid Bell measurement for entanglement swapping (Fig. 8) means projecting subsystems 2 (the first qubus mode) and 3 (the second qubit) of the initial pair of entangled qubit-qubus states,

$$
\left(|0\rangle|\alpha\rangle_{12}+|1\rangle\left|\alpha e^{i \theta}\right\rangle_{12}\right) \otimes\left(|0\rangle|\alpha\rangle_{34}+|1\rangle\left|\alpha e^{i \theta}\right\rangle_{34}\right) / 2,
$$

onto the Bell basis in Eq. (25). According to the method described in the preceding paragraph, the interaction between the qubit (system 3) and the qubus (system 2) leads to

$$
\begin{aligned}
& \left(|0, \alpha, 0, \alpha\rangle+\left|1, \alpha, 1, \alpha e^{i \theta}\right\rangle\right. \\
& \left.\quad+\left|0, \alpha e^{-i \theta}, 1, \alpha e^{i \theta}\right\rangle+\left|1, \alpha e^{i \theta}, 0, \alpha\right\rangle\right) / 2 .
\end{aligned}
$$

When the first qubus mode (system 2) is unambiguously determined to be in the state $|\alpha\rangle$, a Hadamard gate on the second qubit (system 3) plus measurement in the computational basis yields one of two possible hybrid Bell states for the first qubit (system 1) and the second qubus mode (system 4), with the phase depending on the measurement result. In order to obtain any one of the four hybrid Bell states of Eq. (25), in addition, the first qubus mode (system 2) must be coherently projected onto the subspace corresponding to $\left\{\left|\alpha e^{i \theta}\right\rangle,\left|\alpha e^{-i \theta}\right\rangle\right\}$ (for example, via USD and photon-number resolving detectors).

In the above entanglement swapping scheme, clearly the most practical choice is either $p$ homodyne measurement or USD measurement of the unrotated coherent state. A success probability below $1 / 2$ does not automatically render this scheme inferior to the conventional, deterministic entanglement swapping with the two-qubit entangled states, because in the latter case, first the entanglement needs to be distributed probabilistically with a success probability of at most $1 / 2$ using either $p$ homodyne or USD measurements. This probabilistic element is now simply incorporated into the hybrid entanglement swapping protocol. In other words, using the hybrid Bellstate analysis, two initial entanglement distributions and subsequent qubit entanglement swapping can be done almost in one go (when the final hybrid Bell state is again converted into a two-qubit entangled state through another CQED interaction and selective measurements with probability 1/2). However, the Bell-state analysis for the two-qubit entangled states in the conventional protocol relies upon complicated two-qubit quantum logic gates; realizable, for instance, using another four CQED-based dispersive interactions [19]. The hybrid Bell-state analysis here would not require any extra dispersive interactions in addition to those for the initial entanglement distributions. However, a drawback is that we cannot efficiently purify the hybrid entangled states (as discussed in the preceding section) in order to combine sequences of hybrid entanglement swapping steps with hybrid entanglement purification steps.

\section{CONCLUSION}

In summary, we investigated the protocol for a hybrid quantum repeater, based upon dispersive light-matter interactions between electronic spins and bright coherent 
light, with respect to the different kinds of entangled states at the intermediate steps of the protocol and with regard to the final optical measurements for conditionally preparing two-qubit entangled states. As an alternative detection scheme, we propose to apply USD-based measurements on the optical qubus modes.

Compared to the homodyne-based scheme, there are various advantages of the USD-based protocol. First of all, one source of errors can be completely eliminated from the protocol, namely those errors arising from the inability of perfectly discriminating phase-rotated coherent states in phase space. In the USD scheme, this imperfection only leads to smaller efficiencies for the entanglement generation, but the fidelities are unaffected. As a result, the fidelities are solely degraded through the decoherence effect caused by photon losses in the communication channel. By choosing weaker coherent states, the decoherence effect can be suppressed and, in principle, repeater spacings of far beyond $10 \mathrm{~km}$ are possible at the expense of smaller entanglement distribution rates. For example, initial fidelities of about 0.7 are achievable over $50 \mathrm{~km}$ and $100 \mathrm{~km}$ with success probabilities of about $1 \%$ and $0.01 \%$, respectively, using simple on-off detectors (discriminating between vacuum and non-vacuum states). The final two-qubit entangled states here, being, in principle, ideal rank two mixtures, can be purified very efficiently. For the USD-based protocol, we also derived ultimate, distance-dependent bounds on the performance of the entanglement generation step in terms of success probabilities and fidelities.

Finally, we examined the entanglement purification and swapping steps for the hybrid repeater protocol from a different perspective. Instead of performing these steps solely on the level of the two-qubit entangled states, we considered purification and swapping with the hybrid entangled states of the atomic qubit and the optical qubus mode. It turns out that entanglement purification is difficult to achieve, unless optical, non-Gaussian gates (such as Hadamard gates acting upon coherent-state superposition states) are available. Hybrid entanglement swapping, however, can be accomplished easily with exactly the same resources as used for the initial entanglement distribution. In fact, the probabilistic entanglement distribution steps can be incorporated into the hybrid entanglement swapping step, leading to the same overall efficiencies as for the deterministic, qubit entanglement swapping requiring complicated, less feasible quantum logic gates. However, a combination of hybrid entanglement swapping with hybrid entanglement purification in a nested repeater protocol would again require optical, non-Gaussian gates.

\section{Acknowledgments}

PvL acknowledges support from NICT in Japan and the Emmy Noether programme of the DFG in Germany. WJM acknowledges the EU programme QAP. KN acknowledges support in part from NICT and MEXT through a Grant-in-Aid for Scientific Research on Priority Area "Deepening and Expansion of Statistical Mechanical Informatics (DEX-SMI)," No. 18079014. NL acknowledges QAP, QuantumWorks, and the Ontario Centres of Excellence.
[1] W. K. Wootters and W. H. Zurek, Nature 299, 802 (1982).

[2] D. Dieks, Physics Letters 92A, 271 (1982).

[3] H.-J. Briegel, W. Dür, J. I. Cirac and P. Zoller, Phys. Rev. Lett. 81, 5932 (1998).

[4] W. Dür, H.-J. Briegel, J. I. Cirac and P. Zoller, Phys. Rev. A 59, 169 (1999).

[5] M. Zukowski, A. Zeilinger, M. A. Horne, and A. K. Ekert, Phys. Rev. Lett. 71, 4287 (1993).

[6] C. H. Bennett, G. Brassard, S. Popescu, B. Schumacher, J. A. Smolin, and W. K. Wootters, Phys. Rev. Lett. 76, 722 (1996).

[7] D. Deutsch, A. Ekert, R. Jozsa, C. Macchiavello, S. Popescu, and A. Sanpera, Phys. Rev. Lett. 77, 2818 (1996).

[8] L. Hartmann et al., Phys. Rev. A 75, 032310 (2007).

[9] L. Childress, J. M. Taylor, A. S. Sørensen, and M. D. Lukin, Phys. Rev. Lett. 96, 070504 (2006).

[10] L. Childress, J. M. Taylor, A. S. Sørensen, and M. D. Lukin, Phys. Rev. A 72, 052330 (2005).

[11] L. M. Duan, M. D. Lukin, J. I. Cirac, and P. Zoller, Nature 414, 413 (2001).

[12] C. Simon et al., Phys. Rev. Lett. 98, 190503 (2007).
[13] Bo Zhao et al., Phys. Rev. Lett. 98, 240502 (2007).

[14] Z.-B. Chen et al., Phys. Rev. A 76, 022329 (2007).

[15] C. W. Chou et al., Nature 438, 828 (2005).

[16] T. Chaneliere et al., Nature 438, 833 (2005).

[17] M. D. Eisaman et al., Nature 438, 837 (2005).

[18] L. Jiang, J. M. Taylor, and M. D. Lukin, Phys. Rev. A 76, 012301 (2007).

[19] P. van Loock, T. D. Ladd, K. Sanaka, F. Yamaguchi, Kae Nemoto, W. J. Munro, Y. Yamamoto, Phys. Rev. Lett. 96, 240501 (2006).

[20] T. D. Ladd, P. van Loock, K. Nemoto, W. J. Munro, and Y. Yamamoto, New J. Phys. 8, 184 (2006).

[21] T. P. Spiller, K. Nemoto, S. L. Braunstein, W. J. Munro, P. van Loock, and G. J. Milburn, New J. Phys. 8, 30 (2006).

[22] P. van Loock, W. J. Munro, Kae Nemoto, T. P. Spiller, T. D. Ladd, S. L. Braunstein, G. J. Milburn, Los Alamos arXive quant-ph/0701057 (2007).

[23] R. Van Meter, T. D. Ladd, W. J. Munro, and Kae Nemoto, Los Alamos arXive quant-ph/0705.4128 (2007).

[24] for example, Wolfgang P. Schleich, Quantum Optics in Phase Space, (Wiley-VCH, Berlin, 2001).

[25] W. K. Wootters, Phys. Rev. Lett. 80, 2245 (1998). 
[26] I. D. Ivanovic, Phys. Lett. A 123, 257 (1987).

[27] D. Dieks, Phys. Lett. A 126, 303 (1988).

[28] A. Peres, Phys. Lett. A 128, 19 (1988).

[29] W. J. Munro, K. Nemoto, and T. P. Spiller, New J. Phys. 7, 137 (2005).

[30] T. Rudolph, R. W. Spekkens, and P. S. Turner, Phys. Rev. A 68, 010301 (2003).

[31] P. Raynal, N. Lütkenhaus, and S. J. van Enk, Phys. Rev. A 68, 022308 (2003).

[32] P. Raynal and N. Lütkenhaus, Phys. Rev. A 72, 022342 (2005).
[33] P. Raynal and N. Lütkenhaus, Phys. Rev. A 76, 052322 (2007).

[34] J.-W. Pan, C. Simon, C. Brukner, and A. Zeilinger, Nature 410, 1067 (2001).

[35] S. J. van Enk, Phys. Rev. A 66, 042313 (2002).

[36] H. Jeong and M. S. Kim, Quantum Information and Computation 2, 208 (2002).

[37] A. P. Lund, T. C. Ralph, and H. L. Haselgrove, Phys. Rev. Lett. 100, 030503 (2008).

[38] T. C. Ralph, Phys. Rev. A 68, 042319 (2003). 